\title{
Performance evaluation of machine
} learning-based infectious screening flags on the HORIBA Medical Yumizen H550 Haematology Analyzer for vivax malaria and dengue fever

\author{
Parag Dharap ${ }^{1}$ and Sebastien Raimbault ${ }^{2 *}$ (i)
}

\begin{abstract}
Background: Automated detection of malaria and dengue infection has been actively researched for more than two decades. Although many improvements have been achieved, these solutions remain too expensive for most laboratories and clinics in developing countries. The low range HORIBA Medical Haematology Analyzer, Yumizen H550, now provides dedicated flags 'vivax malaria' and 'dengue fever' in routine blood testing, developed through machine learning methods, to be used as a screening tool for malaria and dengue fever in endemic areas. This study sought to evaluate the effectiveness of these flags under real clinical conditions.

Methods: A total of 1420 samples were tested using the Yumizen H550 Haematology Analyzer, including 1339 samples from febrile patients among whom 202 were infected with malaria parasites (Plasmodium vivax only: 182, Plasmodium falciparum only: 18, both: 2), 210 were from febrile dengue infected patients, 3 were from afebrile dengue infected patients and 78 were samples from healthy controls, in an outpatient laboratory clinic in Mumbai, India. Microscopic examination was carried out as the confirmatory reference method for detection of malarial parasite, species identification and assessing parasitaemia based on different stages of parasite life cycle. Rapid diagnostic malarial antigen tests were used for additional confirmation. For dengue infection, NS1 antigen detection by ELISA was used as a diagnostic marker.
\end{abstract}

Results: For the automated vivax malaria flag, the original manufacturer's cut off yielded a sensitivity and specificity of $65.2 \%$ and $98.9 \%$ respectively with the ROC AUC of 0.9 . After optimization of cut-off value, flag performance improved to $72 \%$ for sensitivity and $97.9 \%$ specificity. Additionally it demonstrated a positive correlation with increasing levels of parasitaemia. For the automated dengue fever flag it yielded a ROC AUC of 0.82 with $79.3 \%$ sensitivity and $71.5 \%$ specificity.

Conclusions: The results demonstrate a possibility of the effective use of automated infectious flags for screening vivax malaria and dengue infection in a clinical setting.

*Correspondence: sebastien.raimbault@horiba.com

${ }^{2}$ HORIBA Medical, Montpellier, France

Full list of author information is available at the end of the article

\section{Background Malaria}

Malarial diagnosis is routinely performed with the microscopic examination of thick or thin stained blood smears,

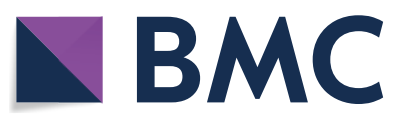

(c) The Author(s) 2020. This article is licensed under a Creative Commons Attribution 4.0 International License, which permits use, sharing, adaptation, distribution and reproduction in any medium or format, as long as you give appropriate credit to the original author(s) and the source, provide a link to the Creative Commons licence, and indicate if changes were made. The images or other third party material in this article are included in the article's Creative Commons licence, unless indicated otherwise in a credit line to the material. If material is not included in the article's Creative Commons licence and your intended use is not permitted by statutory regulation or exceeds the permitted use, you will need to obtain permission directly from the copyright holder. To view a copy of this licence, visit http://creativeco mmons.org/licenses/by/4.0/. The Creative Commons Public Domain Dedication waiver (http://creativecommons.org/publicdomain/ zero/1.0/) applies to the data made available in this article, unless otherwise stated in a credit line to the data. 
a task both time consuming and requiring an experienced microscopist. Immunochromatographic pan-Plasmodium $\mathrm{pLDH}$ antigen detection-based rapid diagnostic tests (RDT) with or without sub-species specific detection system are also used, but are expensive, vulnerable to temperature and humidity as well as demonstrating variable sensitivity and specificity. Other modalities, such as specific PCR [1], flow cytometry-based parasite DNA or RNA detection $[2,3]$ have been developed, but are time consuming, non-affordable, non-automated and therefore are considered impractical for routine application in endemic countries.

\section{Automated malaria detection}

Automated digital microscopy and cell classification with CellaVision (Lund, Sweden) DM96 Advanced RBC Application in the fully automated mode, showed high specificity; however, this has been reported to give a sensitivity of only $23 \%$ [4]. The same image classification software is used in the Sysmex (Kobe, Japan) DI-60, where sensitivity has been found to be similar to microscopy, but only after manual human reclassification [5]. False negative results are still seen, even for $6.3 \%$ parasitaemia, possibly because these systems are very sensitive to smear quality and staining [6]. Furthermore, these systems provide a general parasite class without Plasmodium species identification, again unable to circumvent the need for skilled morphologists.

Haematology Analyzers reporting CBC/DIFF have also been used for malarial screening. Parameters frequently used for screening, such as platelet and WBC counts, may also be affected in diseases other than malaria and hence provide poor sensitivity and specificity. These reported malarial algorithms still involve some mathematical calculation and require interpretative skills to assess laboratory results.

During intra-erythrocytic stages, a malarial parasite in the RBC digests haemoglobin and releases free haem, which is toxic to cells, further converting it into a birefringent insoluble crystalline form called haemozoin [7]. Abbott (Santa Clara, USA) designed the Cell-Dyn series with a depolarized diffracted light (DLL) detector, therefore malaria cases could be flagged by cells identified as large mononuclear cells with a high depolarized signal due to haemozoin $[8,10]$. This detection method lacks sensitivity for early infection, as significant haemozoin production occurs only with late stages or mature Plasmodium parasite forms. Regarding specificity, false positives have been reported in cases of other parasites, such as filaria [9], or children treated with sulfonamide, as its derivatives form yellow granules that give a similar depolarized signal [10]. Varying performance has been reported among studies [10] and among instruments, depending on laser wavelengths. Detection of haemozoin has also been proposed using a magneto-optical device [11].

In infected red blood cells (iRBCs), the cytoskeleton is remodelled by the Plasmodium [12-14], accompanied by changes in $\mathrm{iRBC}$ membrane properties. Although not yet clearly understood, these changes can result in an increased resistance to lysis for iRBCs, especially those harbouring mature forms of the parasite (late amoeboid, schizonts, gametocytes). These incompletely lysed iRBC may then produce a spurious signal in WBC channels, as a peak on the left of WBC volume histogram or as a separate cluster [15]. Since this process is lysis-dependent, balance alarms are often triggered by differences between WBC counting channels using different lysis methods.

Detecting iRBCs has been used for malaria detection with Sysmex XE [16-18], XN series [19] and Mindray (Shenzhen, China) BC-6800 [20]. This technique lacks sensitivity for low levels of parasitaemia and in early infection, as only mature forms are likely to have remodeled the cytoskeleton sufficiently to increase lysis resistance. Furthermore, while Plasmodium vivax remodelling results in a more flexible (deformable) membrane of the $\mathrm{iRBC}$ in order to avoid splenic entrapment, the presence of Plasmodium falciparum conversely affects iRBC by increasing membrane rigidity [21]. The $P$. falciparum survival strategy is to increase iRBC membrane adherence and sequestration in venules and capillaries [22]. This may explain the scarcity of finding mature forms of $P$. falciparum in venous blood, thus resulting in less sensitivity for those Analyzers. Recently, Sysmex has released the high range XN-30 Analyzer, embedding a $405 \mathrm{~nm}$ violet laser with scattering and fluorescence measurements, that has a dedicated module (for additional cost) for Plasmodium detection and counting with a partial lysis reagent allowing parasites to remain inside iRBCs and nucleic acid staining for labelling parasites DNA, which claims an improved detection limit of 20 parasites/ $\mu \mathrm{L}$ [23]. This device has been evaluated for malaria detection, reporting a $\mathrm{ROC}$ with an $\mathrm{AUC}=0.98$ and achieving $98.7 \%$ sensitivity and $96.5 \%$ specificity when compared with microscopy reference [24, 25].

Another technique was developed using various discriminant factors based on statistical variables of cell population data (CPD) for high range Beckman-Coulter instruments using VCS technology to identify malarial infection. Standard deviations of the volume and conductivity of lymphocytes has been used along with PLT count [26], or the standard deviation of the volume of lymphocytes and monocytes [27]. Using CPD on the Sysmex XN, Buoro et al. found 93\% sensitivity, but employing both techniques, an AUC of 0.96 was achieved with 
sensitivity $100 \%$ and specificity $91 \%$, albeit with a limited study of only 14 positive cases [19].

All of these techniques developed to date have been on high range instruments using laser-technology that are generally not in routine use in endemic regions of developing countries where field laboratories typically have low or middle range instruments, thus leaving a persistent need for affordable screening tools. In a previous work [28], the complete data set was generated using two Haematology Analyzers (ABX Pentra XL80, 5-Diff, HORIBA Medical, Montpellier, France and Microsemi CRP, 3-Diff + CRP, HORIBA Medical, Kyoto, Japan) and explored automated SVM classifiers for malaria with data-mining techniques customized for each instrument. The flags generated by these classifiers were evaluated in a prospective study and showed similar performance to claims for malarial flags or algorithms on high range instruments. For the Micros-EMI-CRP, the inclusion of CRP in the flagging algorithm provided valuable information for malaria screening, increasing sensitivity beyond only the CBC data. Unfortunately, the addition of a CRP value to a malarial flagging algorithm does not allow for a cost-effective malarial screening tool.

\section{Dengue fever}

Dengue virus is an arbovirus transmitted by Aedes mosquitoes and exists as four serotypes, DENV-1 to 4 [29]. Dengue infection can range from simple fever to more severe cases with lethal bleeding tendencies due to thrombocytopenia and in the most serious cases with plasma leakage, referred as severe dengue fever. As opposed to malaria where haemozoin or lysis-resistant iRBCs can be observed, there is no obvious specific cellular signal indicative of dengue virus infection in standard Haematology Analyzers. Only the non-specific host immunologic responses common to many viral infections can be observed.

Some effects on haematology parameters, such as lymphocytes, neutrophils and monocytes, have been reported in dengue infections [30,31], which correlate with the number of days of illness and thus are not so effective for screening in early stages of the disease [32].

Some previous studies have defined certain discriminant factors for dengue screening, such as the one developed using CPD on Beckman-Coulter VCS instruments. A review of literature in relation to discriminating dengue fever from other febrile illness can be found in the references [30]. Algorithms distinguishing malaria and dengue fever from other febrile conditions have been designed using the PLT count [26] or via the percentage of lymphocytes and standard deviation of the conductivity of lymphocytes on the LH750, reporting an AUC of 0.893, also evaluated with an AUC of 0.931 in reference
[15]. Soto et al. reported AUC of 0.76 for dengue factor built from the percentage of monocytes and the standard deviation in the volume of monocytes [33]. The lymph-index, defined as lymphocyte mean volume $\mathrm{x} S \mathrm{SD}$ of lymphocyte volume/lymphocyte mean conductivity, has been designed as a factor for viral infection for the LH750, reporting a potential sensitivity of $71 \%$ and specificity of $78 \%$ for dengue fever detection [34, 35]. Dengue factor and lymph-index factor have also been evaluated with 2 new factors: Monocyte Factor from Mono\% and SD of Monocyte volume, and a new dengue factor mixing monocyte factor, lymph-index and platelet (PLT) count [36], but none of these achieved a ROC AUC greater than 0.70 . A decision tree designed with the same variables with the LH780 has been reported to achieve a sensitivity of $94 \%$ and specificity of $77 \%$ for suspected samples [37]. Two decision trees (one of these only using Haematology Analyzer parameters) for classifying severity of the disease have been proposed [38].

\section{Methods}

The purpose of this study was to evaluate the performance of malaria and dengue suspicion flags incorporated into a new generation of low to middle range Haematology Analyzer, the Yumizen H550 by HORIBA Medical, Montpellier, France. Additionally, the achievable performance using further optimized cut-off values was retrospectively evaluated.

\section{Machine learning predictors}

A definite need for automated screening technology has been identified in areas endemic for malaria and dengue. These are largely located in developing countries which, being extremely cost-sensitive, frequently employ low or mid-range analyzers possessing non-laser technology with limited spectral detection capabilities and reagent complexity. Although these low-cost technologies generate limited information for detection, data acquired in such a Haematology Analyser in a single sample run can still range from a hundred to tens of thousands of variables. This work hypothesized that this data may yet have the potential for building an automated classifier algorithm with help of machine learning methods. In this study, two approaches were used. The first was to build the suspicion flags from the raw acquisition data alone in order to avoid revalidation after a change in a single algorithm. While this approach lacks information, such as CPD requiring prior cell classification, it makes the flags generated independent from other computations.

With the second approach, in order to have greater reproducibility between instruments and reagent batches, the measurements were binned, for example a 
128 channels histogram being converted into 16 channels, each derived by the binning of 8 channels.

A set of 243 samples were tested in India in 2017 using reference methods for the target diagnosis (malaria: RDT + thin smear, dengue: ELISA) and processed in duplicate on the Yumizen H550. Various types of classifiers were evaluated with cross-validation techniques, but random forests (using ranger-ml implementation [39]) were found to perform best for these flags, using 1000 trees per forest. Subsequently, a subset of $n=393$ variables were selected with cross-validation, discarding the variables having no relevant information for malaria nor dengue. Instead of classic random forests, classification models where each node has only $\sqrt{ } n \approx 20$ variables were used, regression models using $n / 3=131$ variables for splitting a node were trained to predict probability scores (range [0-1]) of infection with $P$. vivax (probPV) or dengue (probDengue), in order to ease cut-off tuning (default cut-offs being 0.5). Finally, the models were retrained with the whole set of data before evaluation of the prototype software commenced.

\section{Samples}

EDTA anti-coagulated blood samples were collected from patients in Dharap's Diagnostic Center, Mumbai, India between July and October in 2018. The specimens and data were collected in a coded fashion in accordance with local ethical or IRB guidelines and to national and international standards for conducting clinical studies including 21CFR Parts 50 and 56 and International Conference on Harmonization (ICH) E6 -Good Clinical Practice Consolidated Guideline.

The patients' febrile presentation was included, resulting in a febrile group of 1339 samples, an afebrile dengue positive group of three samples and a control non-febrile group of 78 normal samples, totaling 1420 samples (M/F ratio: $56.2 \% / 43.8 \%$, age range: $1-96$ years, grouped as $[\leq 12$ : Child, $\geq 60$ : Elderly, Adult otherwise]).

Among the 1339 febrile patients samples (age: 1-96, M/F: 57\%/43\%), 202 were malaria parasite-infected (age: 5-78, M/F: $67.3 \%$ / 32.7\%, P. vivax only: 182, P. falciparum only: 18, both: 2), 210 were dengue infected (age: 2-90 years, M/F: 61\%/39\%) and 3 samples were concomitantly infected by dengue and vivax malaria.

Thin and thick blood films were prepared for staining and parasite investigation. All the blood smears were examined using a light microscope (Olympus $\mathrm{CH} 30 \mathrm{i}$ ) by an experienced microscopist blinded to the Analyzer flag results.

Microscopic examination of the stained thin smear was conducted as the reference method for identification of Plasmodium species, parasite stages and assessing parasitaemia which was estimated by counting 2000 RBCs.
Rapid malaria antigen detection test kits (SD Biosensor Healthcare Pvt. Ltd., Gurugram, India) using monoclonal anti-P. falciparum HRP-II $(0.75+/-0.15 \mu \mathrm{g})$ with monoclonal anti- $P$. vivax pLDH $(0.75+/-0.15 \mathrm{ug})$ respectively, were used additionally to detect and confirm the presence of $P$. falciparum \& $P$. vivax related antigens.

For screening and diagnosis of dengue fever, RecombiLISA NS1 Antigen test was carried out, utilizing pairs of specific polyclonal \& monoclonal anti-dengue antibodies of all four serotypes (DEN1, 2, 3, 4) with analytical sensitivity of $0.3 \mathrm{ng} / \mathrm{ml}$ for type $2 \mathrm{NS} 1$ antigen (CTK Biotech, Inc., Poway, Ca USA).

All samples were processed in duplicate (2840 total runs) on the Yumizen H550 instrument, using the manufacturer recommended reagents, calibrator and controls. Analyzer performance was monitored daily using three levels of quality control material. Calibration was completed at the start of the study and confirmed at the conclusion of the study.

Yumizen $\mathrm{H} 550$ is a low range 5-diff automated Analyzer embedding a mini flowcell for WBC counting and differential, in addition to RBC/PLT channels for counting and volume measurement by impedance and a $\mathrm{HGB}$ channel. The WBC channel provides a volume/scattering matrix where a cluster of events can be seen, mainly with mature forms of $P$. vivax (Fig. 1).

\section{Data analysis}

Data analysis was performed using Microsoft Office Excel. Statistical analysis was performed using AnalyseIt 4.65.2 (Analyse-it Software Ltd, UK) for Excel. ROC analysis with Wilcoxon-Mann-Whitney AUC estimator and Delong et al. $95 \% \mathrm{CI}$, sensitivity and specificity with Wilson 95\% CI and predictive values with Mercado-Wald

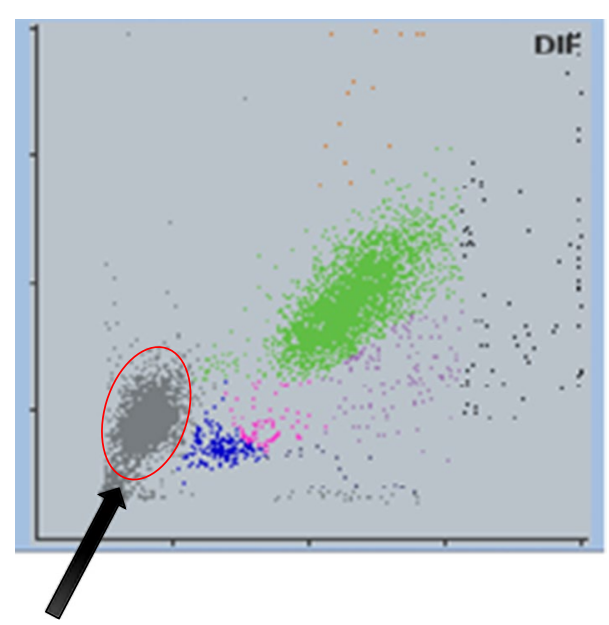

Fig. 1 Cluster of iRBCs containing malarial forms in WBC matrix 

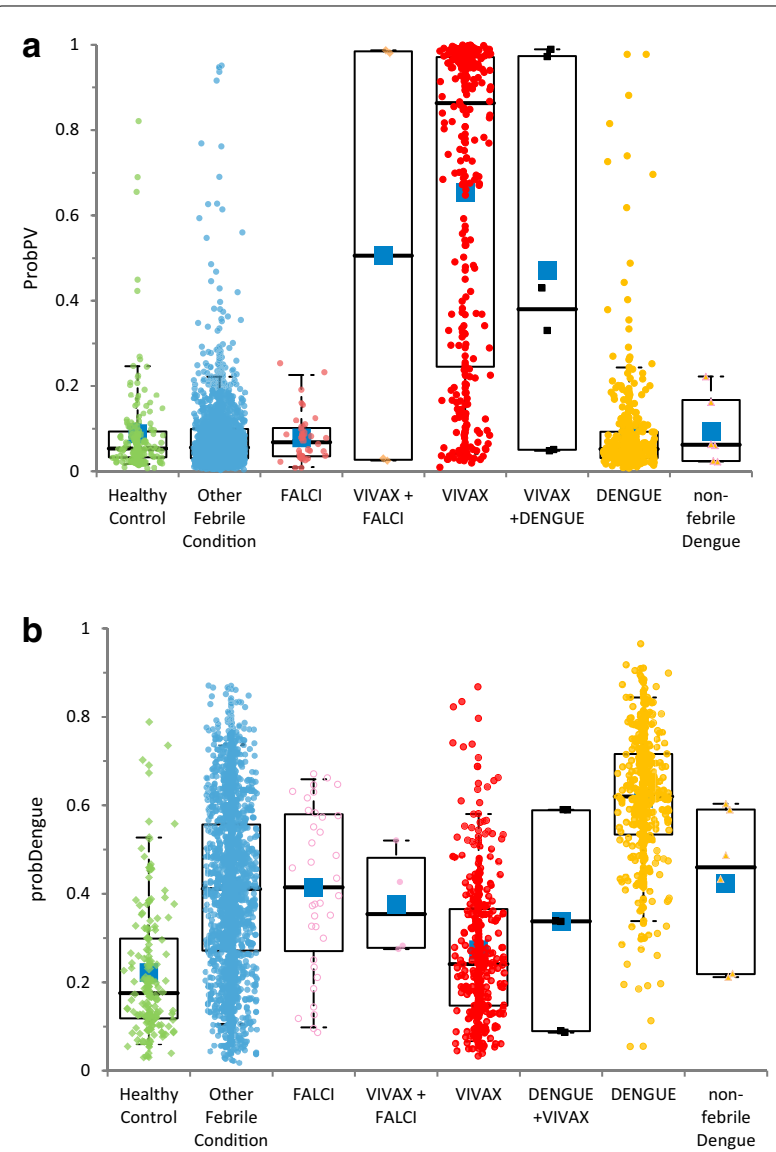

Fig. 2 Distribution of scores for all groups. a ProbPV. b ProbDengue. Box plot legend: plain box: q1-q3 quartiles, dash-line: 5-95\% quantiles, bold line: median, blue square: mean
95\% CI for the detection of $P$. vivax or dengue fever were calculated.

\section{Results}

Figure 2 shows the distribution for each group of computed scores probPV (vivax) and probDengue. Mean absolute difference between duplicates on [0-1] scale were $0.0277(95 \%<0.10)$ and $0.0445(95 \%<0.13)$, respectively. The ROCs of first and second replicates showed no significant difference for each score.

Table 1 shows the performance of the flags for all samples, for febrile patients, for malaria and/or dengue positive patients, and finally for malaria positive patients in order to evaluate $P$. vivax identification ability of probPV. The possible performance with a retrospectively optimized vivax malaria flag cut-off of 0.31 is also presented.

\section{Vivax malaria flag}

Some difference in performance between genders was found. (M/F samples: 799/621, vivax malaria positive ratio $15.8 \% / 10.0 \%$ ). ROC AUC are 0.904 for males and 0.895 for females. The following findings support the hypothesis that this might be linked to differences in parasitemia $(\mathrm{M} / \mathrm{F}$ : mean $=4.37 / 3.66$, median $=2.5 / 2.0$, per 1000 RBCs).

Sensitivity was better for males than females (M/F prospective cut-off $0.5: 68.9 \% / 58.1 \%$, retrospectively optimized cut-off 0.31: 75.0\%/66.1\%). ProbPV scores means and medians were higher for vivax positive males (M/F: $\mathrm{N}=240 / 124$, mean $=0.68 / 0.59$, median $=0.90 / 0.76$ ).

Table 1 Diagnostic performance of vivax malaria and dengue fever flags

\begin{tabular}{|c|c|c|c|c|c|c|}
\hline & & & Overall & Febrile & Malaria or dengue infected & Malaria infected \\
\hline Nsamples & & & 1422 & 1341 & 412 & 202 \\
\hline \multirow[t]{11}{*}{ Vivax malaria } & ROC AUC & & 0.902 [0.880-0.924] & 0.902 [0.880-0.924] & $0.900[0.877-0.923]$ & $0.901[0.868-0.933]$ \\
\hline & cut-off 0.5 & Sensitivity & $65.2 \%[60.2-69.9 \%]$ & $65.2 \%[60.2-69.9 \%]$ & $65.2 \%[60.2-69.9 \%]$ & $65.2 \%[60.2-69.9 \%]$ \\
\hline & & Specificity & $98.9 \%$ [98.5-99.3\%] & $99 \%$ [98.5-99.3\%] & $98.2 \%$ [96.6-99.1\%] & $100 \%[90.4-100 \%]$ \\
\hline & & PPV & $90.2 \%[86.2-93.2 \%]$ & $91.3 \%$ [87.3-94.0\%] & $96.8 \%[93.8-98.4 \%]$ & $100 \%[-]$ \\
\hline & & NPV & $95 \%[94.3-95.6 \%]$ & $94.7 \%$ [94.0-95.4\%] & $77.8 \%[75.3-80.1 \%]$ & $22 \%[19.6-24.4 \%]$ \\
\hline & & Younden & 0.642 & 0.642 & 0.635 & 0.652 \\
\hline & cut-off 0.31 & Sensitivity & $72.0 \%[67.2-76.4 \%]$ & $72.0 \%[67.2-76.4 \%]$ & $72.0 \%[67.2-76.4 \%]$ & $72.0 \%[67.2-76.4 \%]$ \\
\hline & & Specificity & $97.9 \%[97.3-98.4 \%]$ & $98 \%$ [97.4-98.5\%] & $96.9 \%[94.9-98.2 \%]$ & $100 \%[90.4-100 \%]$ \\
\hline & & PPV & $83.9 \%[79.7-87.3 \%]$ & $85.2 \%[81.1-88.5 \%]$ & $95.0 \%$ [91.8-97.0\%] & $100 \%[-]$ \\
\hline & & NPV & $95.9 \%$ [95.2-96.5\%] & $95.6 \%$ [94.9-96.3\%] & $81.1 \%$ [78.4-83.5\%] & $25.9 \%[22.9-29.2 \%]$ \\
\hline & & Younden & 0.699 & 0.700 & 0.689 & 0.720 \\
\hline \multirow[t]{6}{*}{ Dengue } & ROC AUC & & $0.814[0.793-0.834]$ & 0.808 [0.787-0.829] & $0.904[0.883-0.926]$ & - \\
\hline & cut-off 0.5 & Sensitivity & $79.3 \%$ [75.2-82.9\%] & $80.0 \%$ [75.9-83.5\%] & $79.3 \%$ [75.2-82.9\%] & - \\
\hline & & Specificity & $71.6 \%$ [69.8-73.3\%] & $70.1 \%$ [68.1-71.9\%] & $86.4 \%$ [82.7-89.5\%] & - \\
\hline & & PPV & $33.0 \%$ [31.3-34.8\%] & $33.2 \%$ [31.5-35.0\%] & $86.2 \%$ [82.9-89.0\%] & - \\
\hline & & NPV & $95.2 \%[94.2-96.0 \%]$ & $95.0 \%$ [93.9-95.8\%] & $79.6 \%$ [76.4-82.5\%] & - \\
\hline & & Younden & 0.509 & 0.501 & 0.658 & - \\
\hline
\end{tabular}


Some differences were also seen depending on age (Fig. 3). The age groups had different sizes (Child: 165, Adult: 1075, Elderly: 180) and vivax positive ratios (Child: 3.03\%, Adult: $13.3 \%$, Elderly: 20\%).

Performance was lower for children than for adults, but this is likely a function of the limited number of samples, with only 5 vivax positive children, among which 2 with high parasitemia were detected and 3 with low parasitemia were not detected. ROC for group Elderly showed AUC slightly lower than for adults, although parasitemia was marginally higher for this group.

Performance of Pv detection was clearly linked to parasitaemia level. Plasmodium vivax positive samples were classed into 3 parasitaemia groups. For the low parasitaemia group $(\mathrm{p}<0.1 \% \mathrm{RBC}, \mathrm{N}=64)$, sensitivity with default cut-off 0.5 was only $28.9 \%$ and $35.9 \%$ with optimized cut-off 0.31 . For the medium parasitaemia group $(0.1 \% \mathrm{RBC}<\mathrm{p}<1 \% \mathrm{RBC}, \mathrm{N}=97)$, sensitivity with default cut-off 0.5 was $80.9 \%$ with default cut-off and $89.2 \%$ with an optimized cut-off. For the high parasitaemia group ( $\mathrm{p}>1 \% \mathrm{RBC}, \mathrm{N}=23$ ), sensitivity was $100 \%$ with both cutoffs. Figure 4 a shows the values of probP $V$ for vivax positive samples among these groups.

The detection rate was also dependent on the predominant forms of the Plasmodium. For rings, sensitivity was $69.8 \%$ with cut-off 0.5 , and $77.5 \%$ with optimized cut-off 0.31 . For amoeboid trophozoites, sensitivity was only $47.2 \%$ with cut-off 0.5 , and $53.6 \%$ with optimized cut-off 0.31 . For schizonts, sensitivity was $74.6 \%$ with cut-off 0.5 , and $80.3 \%$ with optimized cut-off 0.31 . Figure $4 \mathrm{~b}$ shows the values of probPV for vivax positive samples among these groups. The detection rate was much better for schizonts, as these mature forms typically produce more lyse resistance and thus give a stronger signal in the iRBC cluster of the WBC matrix (Fig. 1). However, it is unclear why detection rate was better when the predominant form was ring rather than amoeboid.

\section{Dengue fever flag}

Some difference in performance associated with gender was also found among the dengue fever patients (F/M samples: 621/799, dengue fever positive ratio 13.4\%/16.3\%) (Fig. 5a). ROC AUC were 0.802 for females and 0.821 for males (Fig. 5a). Sensitivity was slightly better for males $(79.6 \%)$ than females $(78.9 \%)$ with same specificity (71.6\%).

Some differences were also seen depending on age (Fig. 5b). The age groups had different sizes (Child: 165, Adult: 1075, Elderly: 180) and dengue fever positive ratios (Child: $18.5 \%$, Adult: 16.4\%, Elderly: 3.3\%). For the paediatric group, the lowest specificity was observed. For elderly group, there was a limited number of positive
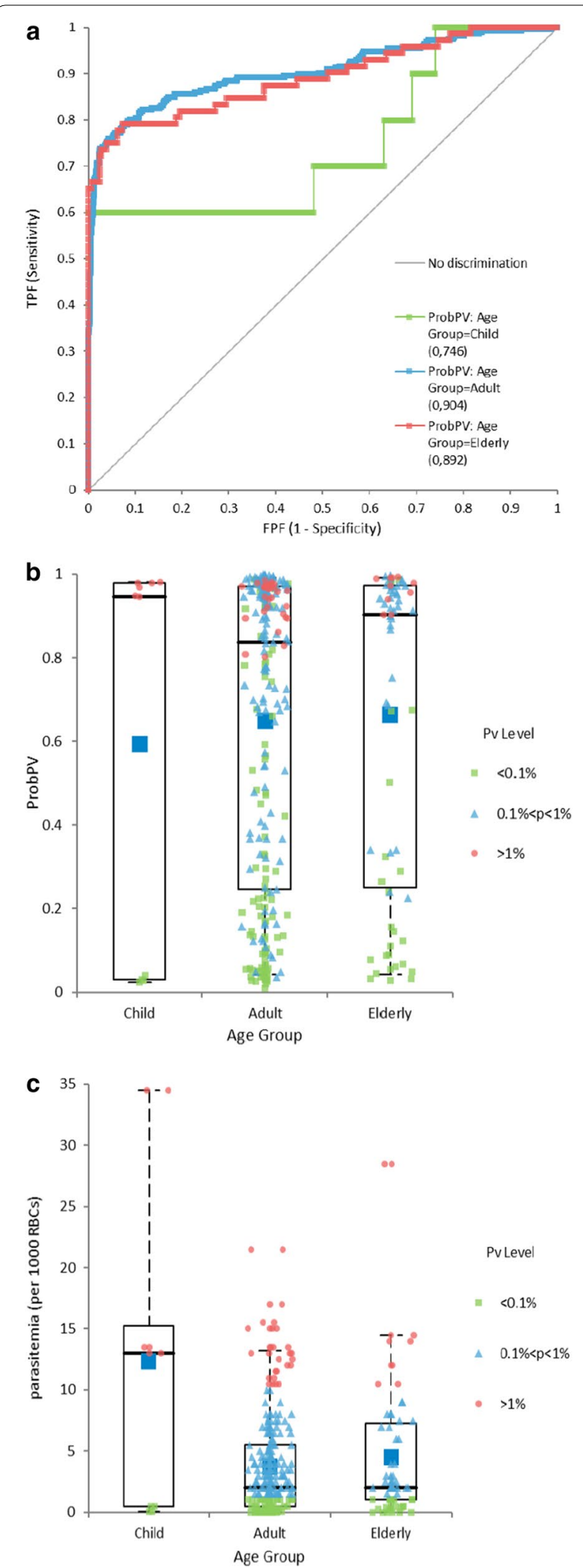

Fig. 3 Vivax detection by age group. a ProbPV ROCs by age group. b Vivax positive ProbPV values by age group. c Vivax positive parasitaemia by age group 


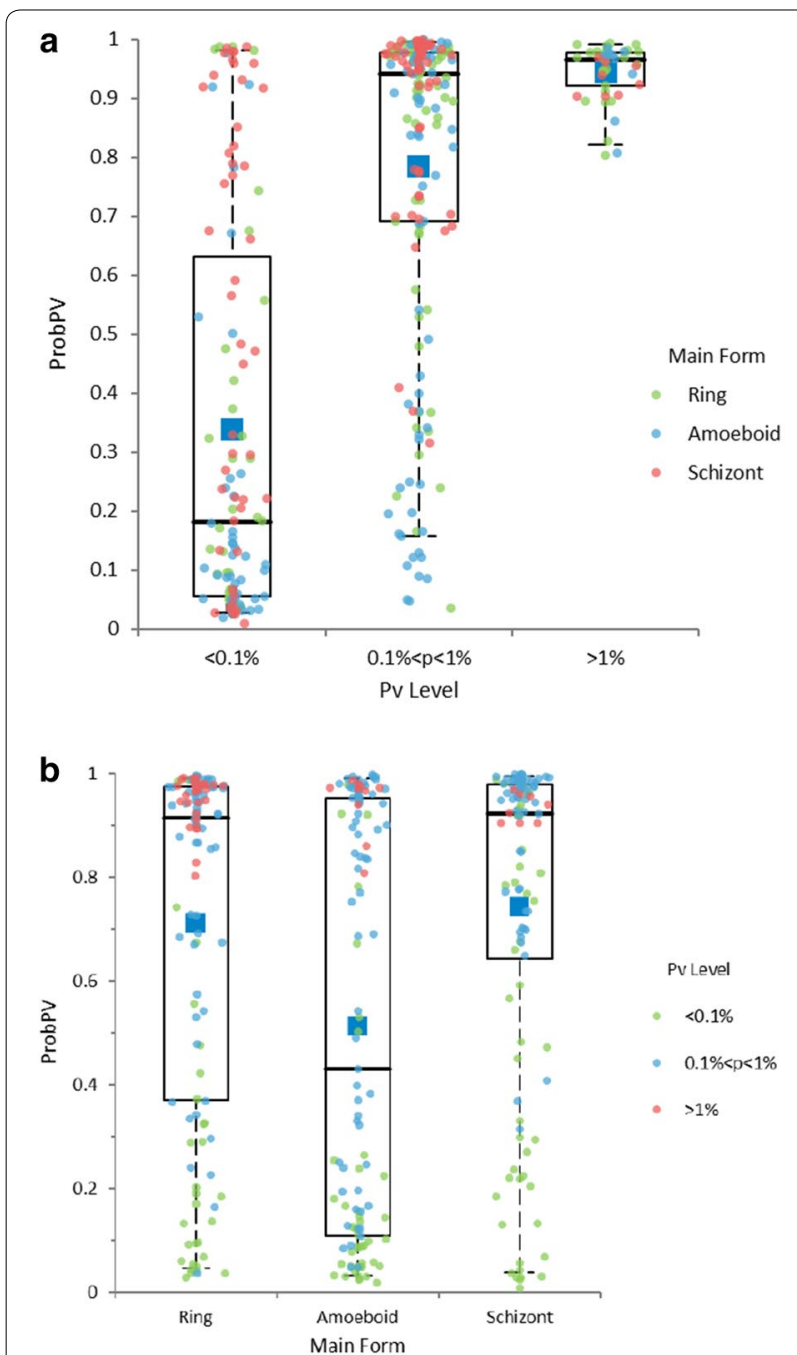

Fig. 4 Vivax detection by parasitaemia and predominant form. a Vivax positive ProbPV values by parasitaemia groups. $\mathbf{b}$ Vivax positive ProbPV values by predominant form

samples (6 so only 12 runs), but probDengue values were lower (Fig. 5c).

\section{Discussion}

The malaria cases presenting with abnormal scatter events as an iRBC cluster of the WBC matrix (Fig. 1) were further investigated to correlate with the presence of specific malarial parasite forms. The size of these clusters was computed from the proportion of cellular events for $100 \mathrm{WBCs}$ and WBC counts, and correlated with the microscopic counts of malarial parasite forms per 2000 RBCs extrapolated for the RBC counts from the Analyzer. No correlation was found for ring forms nor amoeboid forms of Plasmodium with the iRBC counts. However, a strong correlation $\left(R^{2}=0.893\right)$ was found between the
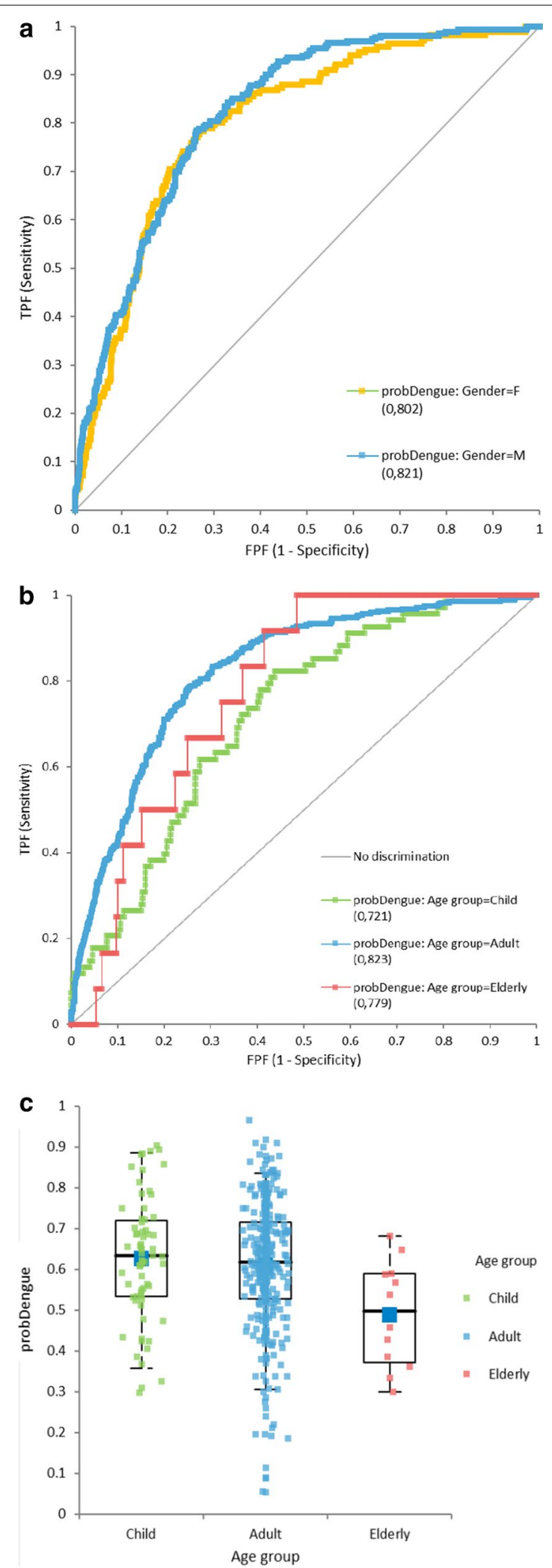

Fig. 5 Dengue detection by gender and age group. a ProbDengue ROCs by gender. b ProbDengue ROCs by age group. c Dengue positive ProbDengue values by age group 
most mature forms of the Plasmodium (schizonts and gametocytes) and these iRBC events in the WBC matrix (Fig. 6a).

This suggests that only these mature forms are large enough to be detected by the optical plus impedance technology of the Yumizen H550. It is also possible that these mature forms have remodeled the iRBC cytoskeleton resulting in increased lyse resistance. Surprisingly, the slope of linear model was about 0.2 , suggesting that only $20 \%$ of these mature forms are seen in the WBC matrix and suggesting only a subset of parasitized RBCs are lyse resistant.

A few samples showed two distinct clusters of iRBCs (Fig. 6b). In some of these samples, the upper right cluster was higher in density than the lower left one, ruling out doublets to be the cause. Neither platelet clumps nor macro-platelets were seen on the corresponding slides.
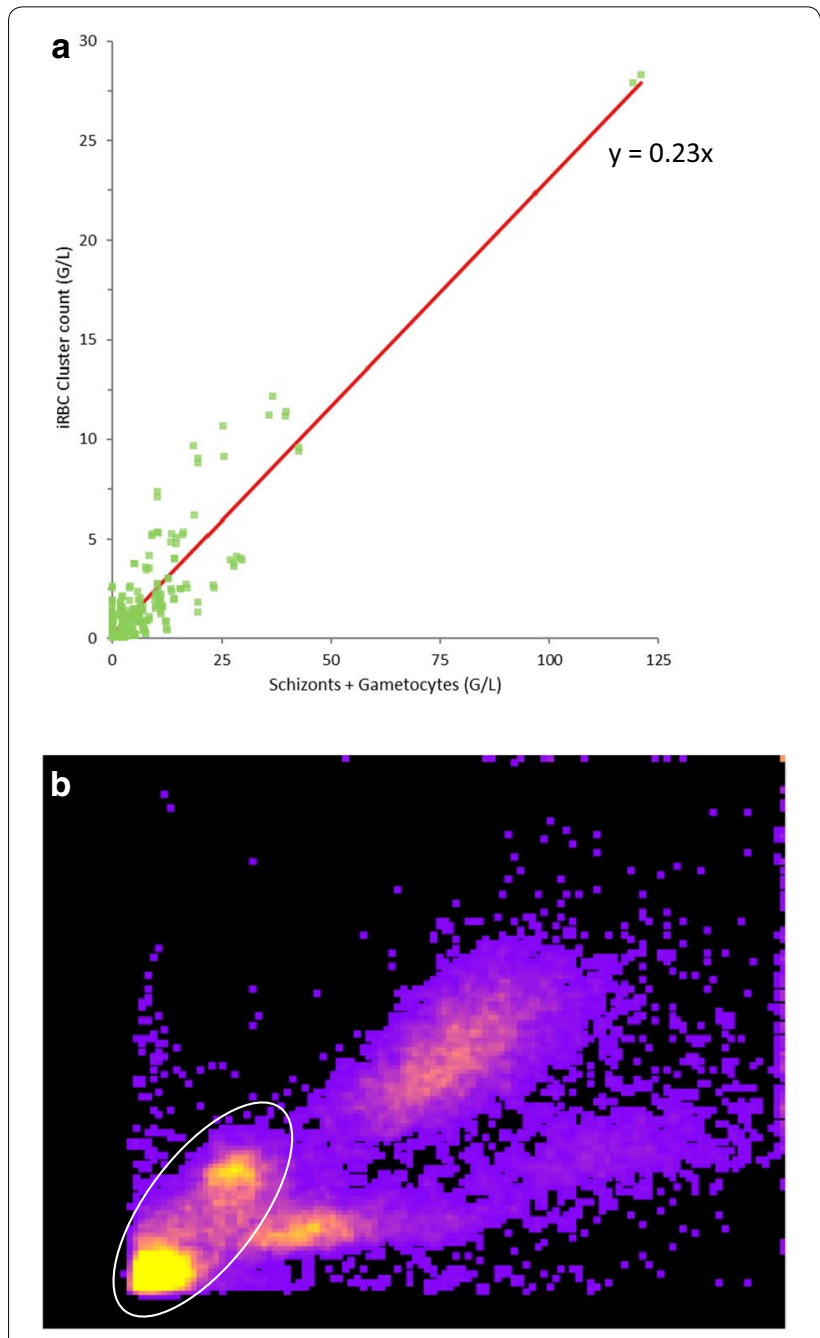

Fig. 6 a Correlation of iRBC cluster counts vs microscopic schizonts + gametocytes counts. b Screen shot of density plot showing double iRBCs clusters seen in a subset of malarial cases
This was also observed in some samples where the predominant forms were rings and schizonts. Presently the exact interpretation of these two clusters remains unclear and a subject of further study.

Although the dengue fever flag performance was lower than that for the vivax malaria flag, it is worthwhile to note that currently there is no reported specific pattern of dengue infection by any standard commercial Haematology CBC-DIFF Analyzer. The machine learning process however was able to build a model performing a relatively efficient screening indicator for dengue infection. This suggests that the machine learning process has extracted many subtle but parallel variations in cell measures resulting from the unique immune response of patients with dengue fever. These findings suggest further investigative 'profiling' of various other viral diseases might be equally productive.

These findings are very encouraging for the feasibility of an affordable commercial malaria and dengue infection screening flag on cost-effective Haematology Analyzers, but still represent a single site study of an ethnically-biased population with a predominance of one malarial species. Profiling the immune response of the patient might also lead to diagnostic pitfalls or limitations, because the response may not be universal and vary from location to location depending on the ethnicities and genetics of patients and/or genetic variations of pathogens. Another limitation of this study is that the performance evaluation was carried out at a single location with a limited number of instruments, albeit that the same instrument was not used to build the models for the malarial and dengue fever flags as that used in the initial performance evaluation.

\section{Conclusions}

For the first time with Haematology Analyzers using cost-efficient technology, the HORIBA Medical Yumizen H550 demonstrates flags for vivax malaria and dengue fever that can be clinically useful for the screening of these infections in low-resourced, endemic areas and thus facilitate further diagnosis testing in a cost effective manner.

Unlike previously reported malarial and dengue screening studies using only high range instruments [7-9, 16-20, 23-27], this approach is based on machine learning from instrument generated raw data measurements from a more affordable CBC-DIFF analysis and does not depend on prior cell population classifications of the sample. This ability to screen for diseases like dengue fever without a unique disease specific signal suggests that machine learning data-mining techniques can provide means of disease profiling based upon the immune or cellular responses of the patient. This approach with 
continued refinement could be extended to screen for various other pathologies and performance could be further improved as the experiential database increases.

\begin{abstract}
Abbreviations
RBC: Red blood cell; iRBC: Infected red blood cell; CBC: Complete blood count; DIFF: Leucocytes differential; WBC: White blood cell; ROC: Receiver operating characteristic; AUC: Area under curve; Cl: Confidence interval; PLT: Platelets; PPV: Positive predictive value; NPV: Negative predictive value; RDT: Rapid diagnostic test; CRP: C-reactive protein.
\end{abstract}

\section{Acknowledgements}

The authors thank Bruce H. Davis, MD, and Mandy Campbell for manuscript review and for their general advice. The machine learning flags had been designed in collaboration with IMAG (Institut Montpelliérain Alexander Grothendieck), University of Montpellier, France.

\section{Author's contribution}

PD was the Principal Investigator of the study, performed patient inclusion, diagnostic, samples processing, data collection and co-wrote the manuscript. SR created machine-learning flags previous to the study, performed data management and statistical analysis, defined a retrospectively optimized cut-off for vivax malaria flagging and co-wrote the manuscript.

\section{Funding}

HORIBA Medical sponsored the study by providing a test instrument with reagents and paying for additional reference tests on samples.

\section{Availability of data and materials}

The datasets used and/or analyzed during the current study are available from the corresponding author on reasonable request.

\section{Competing interest}

Parag Dharap has been a Medical Evaluator for HORIBA Medical since 2018 Sebastien Raimbault is an employee of HORIBA Medical.

\section{Ethics approval and consent to participate}

This study, registered as reference IEC / 19 / 01, has received approval on 6th June 2019 for retrospective analysis from INDEPENDENT ETHICS COMMITTEE (IEC), Reg No. 1433 / 1999 G.B.B.S.D., ETHICS COMMITTEE REGISTRATION No. ECR / 196 / Indt / MH / 2014 issued under Rule 122 DD of Drugs \& Cosmetics Rule 1945. Waiver of Informed consent had been requested and has been granted as protocol was meeting the following criteria: The research presents no more than "minimal risk" of harm to subjects. The waiver or alteration will not adversely affect the rights and welfare of the subjects. The research could not practicably be carried out without the waiver or alteration. The results of instrument generated suspect flags, though indicate the possibility of existence of an abnormality, are only for the technical assistance of laboratory personnel \& are never directly conveyed to the clinician or patient without verification. Therefore, they do not have impact on the referring clinician's judgment or on the patient's state of mind.

\section{Consent for publication}

Not applicable.

\section{Author details \\ ${ }^{1}$ Dharap's Diagnostic Center, Mumbai, India. ${ }^{2}$ HORIBA Medical, Montpellier, France.}

Received: 27 March 2020 Accepted: 16 November 2020

Published online: 23 November 2020

\section{References}

1. Zalis MG, Ferreira-da-Cruz MF, Balthazar-Guedes HC, Banic DM, Alecrim W, Souza JM, et al. Malaria diagnosis: standardization of a polymerase chain reaction for the detection of Plasmodium falciparum parasites in individuals with low-grade parasitemia. Parasitol Res. 1996;82:612-6.

2. Grimberg B, Erickson J, Sramkoski R, Jacobberger J, Zimmerman P. Monitoring Plasmodium falciparum growth and development by UV flow cytometry using an optimized Hoechst-Thiazole orange staining strategy. Cytometry A. 2008;73:546-54.

3. Wongchotigul V, Suwanna N, Krudsood S, Chindanond D, Kano S, Hanaoka N, et al. The use of flow cytometry as a diagnostic test for malaria parasites. Southeast Asian J Trop Med Public Health. 2004;35:552-9.

4. Yoon J, Kwon JA, Yoon SY, Jang WS, Yang DJ, Nam J, et al. Diagnostic performance of CellaVision DM96 for Plasmodium vivax and Plasmodium falciparum screening in peripheral blood smears. Acta Trop. 2019;193:7-11.

5. Racsa LD, Gander RM, Southern PM, McElvania TeKippe E, Doern C, et al. Detection of intracellular parasites by use of the CellaVision DM96 analyzer during routine screening of peripheral blood smears. J Clin Microbiol. 2015;53:167-71.

6. Park M, Hur M, Kim H, Kim HN, Kim SW, Moon HW, et al. Detection of Plasmodium falciparum using automated digital cell morphology analyzer Sysmex DI-60. Clin Chem Lab Med. 2018;56:e284-328.

7. Lawrence C, Olson JA. Birefringent hemozoin identifies malaria. Am J Clin Pathol. 1986;86:360-3.

8. Mendelow BV, Lyons C, Nhlangothi P, Tana M, Munster M, Wypkema E, et al. Automated malaria detection by depolarization of laser light. Br J Haematol. 1999;104:499-503.

9. Crespo S, Palacios G, Scott S, Lago M, Puente S. Abnormal depolarizing patterns in three patients with filarial infection. Ann Hematol. 2004;83:313-5.

10. Baig MA. Evaluation of false + ve cases \& diagnostic accuracy of Abbotts CELL-DYN RUBY for diagnosis of malaria parasite. Int J Sci Res. 2015;4:1232-4.

11. Orbán Á, Butykai Á, Molnár A, Pröhle Z, Fülöp G, Zelles T, et al. Evaluation of a novel magneto-optical method for the detection of malaria parasites. PLoS ONE. 2014;9:e96981.

12. Maier A, Rug M, O'Neill MT, Brown M, Chakravorty S, SzestakT, et al. Exported proteins required for virulence and rigidity of Plasmodium falciparum-infected human erythrocytes. Cell. 2008;134:48-61.

13. Rug M, Cyrklaff M, Mikkonen A, Lemgruber L, Kuelzer S, Sanchez CP, et al. Export of virulence proteins by malaria-infected erythrocytes involves remodeling of host actin cytoskeleton. Blood. 2014;124:3459-68.

14. Glenister FK, Coppel RL, Cowman AF, Mohandas N, Cooke BM. Contribution of parasite proteins to altered mechanical properties of malariainfected red blood cells. Blood. 2002;99:1060-3.

15. Sharma P, Bhargava M, Sukhachev D, Datta S, Wattal C. LH750 hematology analyzers to identify malaria and dengue and distinguish them from other febrile illnesses. Int J Lab Hematol. 2014;36:45-55.

16. Huh HJ, Oh GY, Huh JW, Chae SL. Malaria detection with the Sysmex XE-2100 hematology analyzer using pseudoeosinophilia and abnormal WBC scattergram. Ann Hematol. 2008;87:755-9.

17. Campuzano-Zuluaga G, Alvarez-Sánchez G, Escobar-Gallo GE, ValenciaZuluaga LM, Ríos-Orrego AM, Pabón-Vidal A, et al. Design of malaria diagnostic criteria for the Sysmex XE-2100 hematology analyzer. Am J Trop Med Hyg. 2010;82:402-11.

18. Yoo JH, Song J, Lee KA, Sun YK, Kim YA, Park TS, et al. Automated detection of malaria-associated pseudoeosinophilia and abnormal WBC scattergram by the Sysmex XE-2100 hematology analyzer: a clinical study with 1801 patients and real-time quantitative PCR analysis in vivax malaria-endemic area. Am J Trop Med Hyg. 2010;82:412-4.

19. Buoro S, Manenti B, Seghezzi M, Moili V, Bagorria M, Callegaro A, et al. Abnormal scattergrams and cell population data generated by fully automated hematological analyzers: New tools for screening malaria infection? Int J Lab Hem. 2018;40:326-34.

20. Sun Y, Xiang D, Chen C, He S, Qi H, Wang C. Infected RBC flag/parameter provided by Mindray BC-6800 haematology analyzer aid the diagnosis of malaria. Malar J. 2019;18:262.

21. Suwanarusk R, Cooke BM, Dondorp AM, Silamut K, Sattabongkot J, White $\mathrm{NJ}$, et al. The deformability of red blood cells parasitized by Plasmodium falciparum and P. vivax.J Infect Dis. 2004;189:190-4.

22. Dhangadamajhi G, Kar SK, Ranjit M. The survival strategies of malaria parasite in the red blood cell and host cell polymorphisms. Malar Res Treat. 2010;2010:973094. 
23. Tougan T, Suzuki Y, Itagaki S, Izuka M, Toya Y, Uchihashi K, Horii T. An automated haematology analyzer XN-30 distinguishes developmental stages of falciparum malaria parasites cultured in vitro. Malar J. 2018;17:59.

24. Post A, Kaboré B, Reuling IJ, Bognini J, Van der Heijden W, Diallo S, et al. The $\mathrm{XN}-30$ hematology analyzer for rapid sensitive detection of malaria: a diagnostic accuracy study. BMC Med. 2019;17:103.

25. Pillay E, Khodaiji S, Bezuidenhout BC, Litshie M, Coetzer TL. Evaluation of automated malaria diagnosis using the Sysmex XN-30 analyser in a clinical setting. Malar J. 2019;18:15

26. Jadhav S, Oswal J. Automated cellular indices to identify dengue and malaria and distinguish them from other febrile illnesses. Int J Curr Adv Res. 2018;7:12176-90.

27. Briggs C, Da Costa A, Freeman L, Aucamp I, Ngubeni B, Machin SJ. Development of an automated malaria discriminant factor using VCS technology. Am J Clin Pathol. 2006;126:691-8.

28. Dharap P, Raimbault S, Arnavielhe S, Dray G, Janaqi S, Plantie M, et al. Validation of HORIBA Medical Pentra 80XL/XLR and MicrosemiCRP malaria flag performance derived from algorithmic data-mining techniques. Int J Lab Hem. 2017;39(suppl. 2):33.

29. Henchal EA, Putnak JR. The dengue viruses. Clin Microbiol Rev. 1990;3:376-9.

30. Potts JA, Rothman AL. Clinical and laboratory features that distinguish dengue from other febrile illnesses in endemic populations. Trop Med Int Health. 2008;13:1328-40.

31. Castillo JA, Naranjo JS, Rojas M, Castaño D, Velila PA. Role of monocytes in the pathogenesis of dengue. Arch Immunol Ther Exp. 2019;67:27-40.

32. Chaloemwong J, Tantiworawit A, Rattanathammethee T, Hantrakool S, Chai-Adisaksopha C, Rattarittamrong E, et al. Useful clinical features and hematological parameters for the diagnosis of dengue infection in patients with acute febrile illness: a retrospective study. BMC Hematol. 2018;18:20.

33. Soto R, Irizzary F. Combination of quantitative and morphologic monocyte parameters enhances the performance of the Cbc-differential in the detection of dengue fever. Int J Lab Hem. 2011;33(Suppl. 1):Abstract 145.

34. Zhu Y, Cao X, Tao G, Xie W, Hu Z, Xu D. The lymph index: a potential hematological parameter for viral infection. Int J Infect Dis. 2013;17:e490-3.

35. Hawaldar R, Sodani S. Evaluation of LH 750 VCS parameters and lymph Index in identifying Dengue fever. Indian J Pathol Oncol. 2015;2:76-80.

36. Jaykar HH, Kelkar AJ, Mani NS. Applicability of volume conductivity and scatter parameters for early prediction of dengue virus infection. J Appl Hematol. 2018;9:1-4.

37. Justin J, Suman FR, Sukhachev D, Naveen K, Rajendran R, Lakshmi U. Utility of cell population data as an early predictor of dengue. Saudi J Pathol Microbiol. 2018:3:135-9.

38. Potts JA, Gibbons RV, Rothman AL, Srikiatkhachorn A, Thomas SJ, Supradish $\mathrm{PO}$, et al. Prediction of dengue disease severity among pediatric Thai patients using early clinical laboratory indicators. PLoS Negl Trop Dis. 2010;4:e769.

39. Wright MN, Ziegler A. ranger: a fast implementation of random forests for high dimensional data in C++ and R. J Stat Softw. 2017;77:1-17.

\section{Publisher's Note}

Springer Nature remains neutral with regard to jurisdictional claims in published maps and institutional affiliations.
Ready to submit your research? Choose BMC and benefit from:

- fast, convenient online submission

- thorough peer review by experienced researchers in your field

- rapid publication on acceptance

- support for research data, including large and complex data types

- gold Open Access which fosters wider collaboration and increased citations

- maximum visibility for your research: over $100 \mathrm{M}$ website views per year

At $\mathrm{BMC}$, research is always in progress.

Learn more biomedcentral.com/submissions 